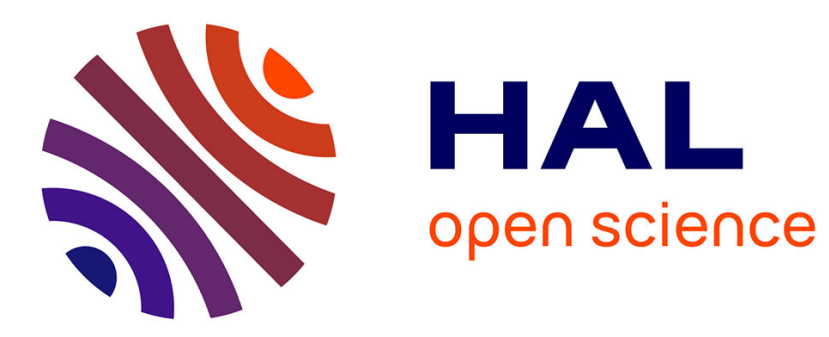

\title{
Gestion de la pandémie COVID-19 en Suisse : rôles et défis d'une pharmacie interhospitalière
}

Laurence Schumacher, Cédric Blatrie, Séverine Krähenbühl, Camilla Pasteur, Anne-Laure Blanc, Charline Pellaton, Pascal Bonnabry, François Rouiller, Nicolas Widmer

\section{To cite this version:}

Laurence Schumacher, Cédric Blatrie, Séverine Krähenbühl, Camilla Pasteur, Anne-Laure Blanc, et al.. Gestion de la pandémie COVID-19 en Suisse: rôles et défis d'une pharmacie interhospitalière. Medecine de Catastrophe Urgences Collectives, 2020, 4 (3), pp.223 - 232. 10.1016/j.pxur.2020.08.010 . hal-03268218

\section{HAL Id: hal-03268218 \\ https://hal.science/hal-03268218}

Submitted on 23 Jun 2021

HAL is a multi-disciplinary open access archive for the deposit and dissemination of scientific research documents, whether they are published or not. The documents may come from teaching and research institutions in France or abroad, or from public or private research centers.
L'archive ouverte pluridisciplinaire HAL, est destinée au dépôt et à la diffusion de documents scientifiques de niveau recherche, publiés ou non, émanant des établissements d'enseignement et de recherche français ou étrangers, des laboratoires publics ou privés. 


\section{Gestion de la pandémie COVID-19 en Suisse : rôles et défis d'une pharmacie interhospitalière}

\section{Management of the COVID-19 pandemic in Switzerland: Roles and challenges of an interhospital pharmacy}

\author{
Laurence Schumacher ${ }^{\text {a,b }}$ \\ Cédric Blatrie ${ }^{b}$ \\ Séverine Krähenbühl ${ }^{\text {b }}$ \\ Camilla Pasteur ${ }^{b}$ \\ Anne-Laure Blanc ${ }^{b}$ \\ Charline Pellaton ${ }^{b}$ \\ Pascal Bonnabry a,c \\ François Rouiller ${ }^{b}$ \\ Nicolas Widmer ${ }^{\mathrm{a}, \mathrm{b}}$
}

\author{
${ }^{a}$ Centre spécialisé de pharmacie d'urgence et de \\ catastrophe, université de Genève, institut des \\ sciences pharmaceutiques de Suisse Occidentale, \\ Genève, Suisse \\ ${ }^{\mathrm{b}}$ Pharmacie des hôpitaux de l'Est lémanique, \\ Rennaz, Suisse \\ ${ }^{\mathrm{C}}$ Pharmacie, hôpitaux universitaires de Genève, \\ Genève, Suisse
}

\section{MOTS CLÉS \\ Plans catastrophe Pharmacie d'hôpital Protection civile Pandémie COVID-19}

\section{KEYWORDS}

Disaster planning Pharmacy Service Hospital Civile Defense Pandemics COVID-19 ques); (4) production et approvisionnement de désinfectant pour les mains ; (5) appui clinique (particulièrement dans les unités de soins critiques); (6) fabrication de médicaments individualisés; (7) gestion des pharmacies d'unité de soins, et (8) fourniture de masques hygiéniques pour les professionnels de la santé de la région. Les missions (4) et (8) ne faisaient pas partie des rôles habituels de la pharmacie et ont été réalisées avec le soutien de la Protection civile suisse. Une difficulté particulière a été la gestion de la pénurie de divers produits et l'identification d'options thérapeutiques alternatives. En conclusion, notre pharmacie d'hôpital a été confrontée à divers défis durant la première vague de la pandémie. Certaines missions réalisées ont même dépassé les responsabilités traditionnelles. Sur la base des enseignements tirés de cette pandémie, le plan d'urgence de notre pharmacie, ainsi que la formation associée du personnel, ont été développés.

(C) 2020 Société Française de Médecine de Catastrophe. Publié par Elsevier Masson SAS. Cet article est publié en Open Access sous licence CC BY-NC-ND (http://creativecommons.org/licenses/by-nc-nd/4.0/).

\section{SUMMARY}

From March 16 to June 18, 2020, because of the COVID-19 pandemic, the Swiss Federal Council declared an "extraordinary situation" in terms of the Epidemics Act. The present work assesses the roles of an interhospital pharmacy in the fight against SARS-CoV-2 in Switzerland, in the frame of the Coordinated Medical Service. During this time, all missions performed by the pharmacy were systematically collected and evaluated. They were also compared to its official duties. Specific missions have been mainly managed by the crisis unit and the 4 departments of

Auteur correspondant : N. Widmer, Pharmacie des hôpitaux de l'Est Lémanique, route du Vieux Séquoia 20, 1847 Rennaz, Suisse.

Adresse e-mail :

nicolas.widmer@phel.ch 
the pharmacy (Logistics, Manufacturing, Clinical Pharmacy and Nursing Homes Supply): (1) guarantee continuity of human resources; (2) internal infrastructure securing (especially in terms of hygiene); (3) specific drug supply (e.g. anesthetics, sedatives, antivirals, incl. for clinical trials); (4) hand disinfectant production and supply; (5) clinical assistance (especially in the Critical Care units); (6) individual drug manufacturing; (7) management of ward pharmacies, and (8) hygienic masks supply for healthcare professionals in the area. The missions (4) and (8) were out of the usual duties of the pharmacy and have been achieved with the support of the Swiss civil protection. A particular difficulty was the management of the shortage of various products and the identification of alternative therapeutic options. In conclusion, our pharmacy has faced various challenges during the first wave of the pandemic. Some missions performed were even beyond the traditional duties. Based on the lessons learned from this pandemic, the disaster plan of our pharmacy, as well as the associated staff training, have been further developed. (c) 2020 Société Française de Médecine de Catastrophe. Published by Elsevier Masson SAS. This is an open access article under the CC BYNC-ND license (http://creativecommons.org/licenses/by-nc-nd/4.0/).

\section{INTRODUCTION}

La Suisse est une confédération composée de 26 cantons et peuplée d'environ 8,5 millions d'habitants. En 2018, la Suisse comptait 281 hôpitaux sur son territoire disposant d'un total de 24226 lits dans des hôpitaux de soins généraux et 13825 dans des cliniques spécialisées [1]. La gestion de la santé en Suisse est principalement répartie entre la Confédération et les cantons, ce qui permet une certaine liberté et explique des différences d'une région à l'autre, y.c. dans le domaine de la gestion des situations d'urgence [2]. Par exemple, dans le canton de Vaud, troisième canton le plus peuplé, la chaîne de secours s'articule autour de trois axes principaux :

- une centrale d'engagement unique pour les appels de demandes sanitaires qui régule les urgences vitales ;

- des secours non médicalisés et ;

- des secours médicalisés.

La centrale d'alarme dispose de moyens sanitaires tels que des ambulances privées ou publiques, des services mobiles d'urgence et de réanimation (SMUR) et un moyen héliporté médicalisé [3] Selon les cantons, les équipages des ambulances d'urgence se composent de un ou deux ambulancier(s) diplômé(s) qui assurent les soins pré-hospitaliers d'urgence $24 \mathrm{~h}$ sur 24 de façon autonome. Dans l'attente de l'arrivée du SMUR, ces professionnels peuvent effectuer des actes médicaux délégués, comme l'administration de médicaments d'urgence, sous la responsabilité d'un médecin conseil (selon protocoles préétablis) [3]. En cas d'accident majeur, les moyens disponibles au quotidien participent en première ligne à la réponse sanitaire, puis ceux-ci bénéficient de moyens additionnels définis par des accords préétablis, comme le poste médical avancé (PMA), des renforts extra-cantonaux ou fédéraux (y.c. militaires) ou même de l'aide de pays voisins, comme la France.

Dans ce contexte, le Service sanitaire coordonné (SSC) a pour objectif de garantir en tout temps et dans toutes les situations une prise en charge sanitaire optimale de tous les patients par une coordination efficace de toutes les organisations partenaires civiles et militaires sur le plan fédéral (partenaires feux bleus, hôpitaux, protection civile, service sanitaire de l'armée) [4]. Pour cela, il élabore avec ses partenaires des recommandations et des formations pour assurer le meilleur niveau de réponse possible. En cas de conflit armé ou de situation particulière ou extraordinaire, comme une épidémie, un organe de coordination spécifique du SSC (OSANC) prend en charge la coordination opérationnelle [4]. En termes de formation et recherche, la Confédération finance aussi une douzaine de centres spécialisés universitaires sous l'égide du Centre de compétences pour la médecine militaire et de catastrophe (Cen comp MMC) [5]. Le but de certains de ces centres spécialisés est d'élaborer une unité de doctrine dans la conduite des évènements majeurs dans le pays [6]. Pour ce qui est de l'approvisionnement en médicaments, le domaine " produits thérapeutiques » de l'Office fédéral pour l'approvisionnement économique du pays (OFAE) veille à ce que les producteurs de médicaments disposent de suffisamment de réserves de médicaments spécifiques en Suisse en cas de pénurie $[7,8]$. Toutefois, il ne s'agit pas formellement d'un organe de conduite en cas de crise dans le domaine de l'approvisionnement en médicaments et dispositifs médicaux. En Suisse, le risque d'être touché par une pandémie avait été identifié comme risque majeur lors de l'élaboration de la dernière cartographie des risques publiée par les autorités à fin juin 2015 [9]. Peu avant, un exercice du Réseau national de sécurité avait été conduit en novembre 2014 (ERNS 14), fondé notamment sur un scénario de pandémie (nouveau virus se répandant en Asie centrale et touchant l'Europe quatre mois plus tard) et avait débouché sur 16 recommandations, déclinées en 42 mesures, visant à améliorer la collaboration entre la Confédération, les cantons et leurs partenaires en cas de crise [10]. Ces recommandations étaient, par exemple, de développer la gestion fédérale des ressources (ResMaB), une structure permettant à la Confédération d'aider rapidement et de manière ciblée les cantons touchés en leur fournissant les ressources manquantes ou encore de renforcer la collaboration entre l'Office fédéral de la santé publique (OFSP) et le SSC pour apporter un soutien aux cantons en cas de pandémie. En 2019, les mesures associées avaient été en bonne partie élaborées, mais pas encore complètement testées [11].

Dans ce contexte, but du présent article est de décrire l'expérience d'une pharmacie interhospitalière suisse spécifique durant la pandémie COVID-19 pour identifier des potentiels d'amélioration dans la gestion pharmaceutique de crises similaires à l'avenir.

\section{LA PANDÉMIE COVID-19 EN SUISSE}

L'année 2020 est marquée par la pandémie COVID-19 qui, comme la peste noire ou la grippe espagnole, restera certainement dans l'histoire [12]. Fin décembre 2019, les premiers cas d'une pneumonie atypique dus à un nouveau coronavirus son rapportés dans la ville chinoise de Wuhan, capitale de la province du Hubei [13]. En février 2020, l'Organisation mondiale de la santé (OMS) dénomme officiellement ce nouveau virus SARS-CoV-2 et la maladie qu'il entraîne COVID-19 pour « Coronavirus Disease 2019 ». Ce nouveau coronavirus est un 
bêta-coronavirus, tout comme le SARS-CoV, avec lequel il partage plus de $70 \%$ d'homologie. Le SARS-CoV, responsable d'une épidémie de pneumonie ayant touché plus de 8000 personnes de 2002 à 2004 et caractérisé par un taux de mortalité d'environ $10 \%$, est aujourd'hui considéré comme éradiqué [14]. Quant au SARS-CoV-2, il semble associé à une mortalité plus faible, mais très variable d'un pays et d'un système de santé à l'autre [15].

Durant l'hiver 2020, de plus en plus de cas d'infection à SARSCoV-2 sont détectés hors de la Chine, dont plusieurs en Europe. Début mars, le nombre de cas augmente en Europe et principalement en Italie, qui représente un nouveau foyer [16]. Le 12 mars 2020, l'OMS déclare l'état de pandémie de COVID-19. La France, quant à elle, enregistre ses premiers cas mi-février et un pic durant la première quinzaine d'avril [17]. Ces évènements ont mobilisé et mobilisent encore de façon majeure les systèmes de santé mondiaux, y.c. les pharmacies hospitalières.

En Suisse, c'est le 25 février 2020 que le premier cas de COVID19 est rapporté au Tessin, canton limitrophe de I'Italie. Le 12 mars 2020, la Suisse est le troisième pays d'Europe touché par le coronavirus par rapport au nombre d'habitants [17]. Le 13 mars, par le biais de l'Ordonnance 2 COVID-19, le gouvernement suisse(Conseil fédéral) annonce des mesures strictes sur l'ensemble du territoire, comme l'interdiction des rassemblements de plus de 100 personnes, la fermeture des écoles et des universités dans tout le pays et l'introduction de contrôles aux frontières [18]. Parallèlement, de nombreux cantons annoncent aussi des restrictions, parfois plus strictes. Puis le 16 mars, le Conseil fédéral déclare l'état de " situation extraordinaire " (au sens de l'article 7 de la Loi sur les épidémies, LEp) [19] et met à jour son ordonnance avec de nouvelles mesures, comme la fermeture des commerces non essentiels (y.c. les restaurants et les établissements de loisirs) ainsi que celle, partielle, des frontières. En même temps, il décide de la mobilisation d'une partie de l'armée pour assister les structures hospitalières civiles et soutenir les autorités dans diverses tâches de sécurité et de logistique. Parallèlement, les cantons mobilisent également de nombreux appelés de la protection civile. La mobilisation de l'armée, surtout des conscrits des troupes sanitaires, a représenté la plus importante mobilisation depuis la Seconde Guerre mondiale en Suisse [20]. Mi-août 2020, le pays comptait plus de 37000 cas de patients testés positifs et près de 2000 décès [21]. À cette date, le canton de Vaud était le plus touché du pays en nombre de décès absolu (425) et occupait le troisième rang en nombre de cas cumulés par habitant (env. 800/100 000 ; après Genève et le Tessin) [21]. Globalement, le système de santé suisse n'a pas été saturé, mais a dû relever de nombreux défis [22]. Le 19 juin 2020, au vu d'une baisse générale du nombre d'infections, le Conseil fédéral a pu lever l'état de « situation extraordinaire », tout en maintenant un état de " situation particulière ".

Au sein des hôpitaux suisses, une réorganisation des services s'est opérée à différents niveaux et le dispositif hospitalier a été considérablement modifié et renforcé. Certains secteurs d'activités cliniques non urgentes ont été réduits au profit de la création de plusieurs unités de médecine spécialisées dans la prise en charge des patients COVID-19, voire d'hôpitaux entiers dédiés (ex : la Carità dans le canton du Tessin ou le site de Bruderholz dans le canton de Bâle Campagne). Le nombre de lits de soins critiques ont été également considérablement augmentés (plus que doublé dans certains cas) en recourant, par exemple, à des blocs opératoires libérés des interventions électives et permettant de pratiquer des prises en charge intensives. Parfois, la réaffectation d'étages entiers en soins intermédiaires a été réalisée. Les urgences pré-hospitalières et hospitalières ont été impliquées en première ligne dans la réponse à cette crise $[23,24]$. Elles ont également dû s'adapter à ce nouveau risque et ont, notamment :

- ajusté les mesures de protection des premiers répondants aux potentiels risques d'expositions (d'autant plus compte tenu des incertitudes de diagnostic et des procédures à risque d'aérosolisation souvent pratiquées, comme la réanimation) ;

- augmenté les capacités d'accueil des urgences et la redistribution du personnel en fonction des besoins ;

- installé des structures de dépistage particulières.

Toutefois, le nombre d'interventions pré-hospitalières et le nombre de consultations aux urgences sans lien avec la pandémie ont globalement diminué durant cette période. Enfin, ces services ont, comme d'autres, dû faire face à des adaptations des mesures rapides et faire preuve de polyvalence et d'une grande flexibilité en raison de l'évolution constante de la situation $[23,24]$.

\section{LA PHARMACIE DES HÔPITAUX DE L'EST LÉMANIQUE}

La Pharmacie des hôpitaux de l'Est lémanique (PHEL) est installée au sein du Centre hospitalier de Rennaz en Suisse (Hôpital Riviera-Chablais, Vaud-Valais ; HRC). Elle est responsable de l'approvisionnement en médicaments et de l'assistance pharmaceutique de cinq hôpitaux d'intérêt public de l'Est du canton de Vaud et du Chablais valaisan, ainsi que de sept établissements sanitaires privés et 15 maisons de retraite médicalisées (établissements médico-sociaux ; EMS) de la même région. À eux tous, ces établissements totalisent plus de 400 lits de soins aigus, environ 300 lits de soins de réadaptation et plus de 900 lits médico-sociaux. La PHEL, comme d'autres pharmacies interhospitalières suisses, a affronté la pandémie COVID-19. Le présent article documente et évalue toutes les missions effectuées par la PHEL de début mars à fin juin 2020 et les compare aux responsabilités qui sont les siennes en temps ordinaire.

Les missions spécifiques à la pandémie ont été principalement gérées par la cellule de crise de la pharmacie et les collaborateurs des quatre secteurs de celle-ci (Logistique pharmaceutique, Fabrication, Pharmacie clinique et Approvisionnement des maisons de retraite médicalisées). Ces missions sont présentées dans le Tableau I.

La cellule de crise était constituée de la direction de la pharmacie, renforcée, et en lien régulier avec la cellule de crise de I'HRC, principal hôpital de soins aigus de la région. Dans ce contexte de pandémie, la cellule de crise de la pharmacie a partiellement déclenché le plan d'urgence de la pharmacie et installé une salle de conduite dans ses locaux (Fig. 1). Un défi principal a été la gestion des pénuries ou des risques de pénurie de divers produits, ainsi que l'identification d'options thérapeutiques alternatives. De façon générale, le Tableau II présente les produits thérapeutiques dont l'approvisionnement devait être garanti durant cette situation extraordinaire, ainsi que les problématiques associées.

L'implication de la pharmacie dans cette crise a été rendue nécessaire par les défis rencontrés par ses partenaires, mais 
Tableau I. Missions effectuées par les différents secteurs de la Pharmacie des hôpitaux de l'Est lémanique durant la première vague de pandémie COVID-19 au printemps 2020.

\begin{tabular}{|c|c|c|}
\hline Mission & Secteur & Exemple de mesures \\
\hline \multirow{6}{*}{$\begin{array}{l}\text { 1. Garantie de la } \\
\text { continuité des ressources } \\
\text { humaines }\end{array}$} & Cellule de crise & $\begin{array}{l}\text { Création d'un plan de continuité des } \\
\text { ressources humaines }\end{array}$ \\
\hline & & $\begin{array}{l}\text { Vision constante de la globalité des } \\
\text { effectifs }\end{array}$ \\
\hline & & $\begin{array}{l}\text { Établissement d'une liste des } \\
\text { fonctions indispensables au } \\
\text { fonctionnement de la pharmacie } \\
\text { ainsi que des responsables et } \\
\text { remplaçants }\end{array}$ \\
\hline & & $\begin{array}{l}\text { Identification des fonctions et des } \\
\text { collaborateurs pouvant réaliser du } \\
\text { télétravail jusqu'à hauteur de } 100 \% \\
\text { de leur taux de travail }\end{array}$ \\
\hline & & $\begin{array}{l}\text { Planification prévisionnelle } \\
\text { (évaluation des risques ainsi que de } \\
\text { leur évolution supposée et de ses } \\
\text { potentielles conséquences au sein } \\
\text { de la pharmacie) }\end{array}$ \\
\hline & & $\begin{array}{l}\text { Demandes d'appui en personnel au } \\
\text { canton (Protection civile) et à la } \\
\text { Confédération (Armée) }\end{array}$ \\
\hline $\begin{array}{l}\text { 2. Sécurisation de } \\
\text { l'infrastructure de la }\end{array}$ & Cellule de crise & $\begin{array}{l}\text { Mise en place et communication } \\
\text { d'une procédure d'hygiène }\end{array}$ \\
\hline
\end{tabular}

pharmacie
Élaboration d'un plan de protection selon les demandes de la Confédération et communication de la procédure

Fabrication

3. Approvisionnement Cellule de crise
spécifique en
médicaments
spécifique en
Mise en place d'une possibilité de fabrication de désinfectants de surface en cas de pénurie

Suivi rapproché de certains stocks de médicaments jugés indispensables et à risque de rupture

Transmission hebdomadaire à la Confédération d'un inventaire des stocks de médicaments jugés indispensables

Logistique pharmaceutique

\section{Gestion des stocks des} médicaments spécifiques

Gestion des pénuries de médicaments spécifiques ; communication des solutions mises en place

Création de nouveaux lieux de stockage pour les unités spécifiques COVID-19

Soutien logistique dans les unités de soins

\section{Responsabilité usuelle} [oui/non/partiellement]

Non (créé spécialement pour la situation)

Partiellement (habituellement, cette responsabilité est déléguée à chaque responsable de secteur)

Non (créée spécialement pour la situation)

Partiellement (télétravail autorisé à maximum $20 \%$ habituellement)

Non (créée spécialement pour la situation)

Non (demandes propres à la situation)

Non (démarche propre à la situation)

Non (démarche propre à la situation)

Non (les désinfectants de surface ne sont habituellement pas gérés par la pharmacie)

Non (habituellement cette responsabilité est déléguée au secteur Logistique pharmaceutique)

Non (mise en place suite à la demande de la Confédération)

Partiellement (habituellement, les médicaments stockés correspondent à un mois d'utilisation ; dans ce cas, les quantités ont été augmentées pour pallier l'augmentation de consommation de ceux-ci)

Partiellement (activité habituelle, mais renforcée et ciblée sur les problématiques rencontrées lors de la pandémie)

Non (mesures spécifiques mises en place pour les nouvelles unités créées ou celles dont l'activité avait été augmentée)

Partiellement (intensifié dans les unités COVID-19) 
Tableau I. Missions effectuées par les différents secteurs de la Pharmacie des hôpitaux de l'Est lémanique durant la première vague de pandémie COVID-19 au printemps 2020 (suite).

\begin{tabular}{ll}
\hline Mission & Secteur \\
\hline & Logistique \\
& pharmaceutique/ \\
& Fabrication \\
& Pharmacie clinique
\end{tabular}

\section{Exemple de mesures}

Gestion pharmaceutique d'une étude clinique de I'OMS (Solidarity)

Identification des traitements utilisés pour traiter les symptômes du COVID-19, notamment aux soins critiques, et ceux à effet anti-SARSCoV-2

Gestion des
maisons de retraite

\section{Approvisionnement en désinfectants pour les} mains

Cellule de crise

Logistique pharmaceutique

Fabrication

5. Appui clinique

Pharmacie clinique

Production de désinfectants pour les
Suivi des consommations par les patients des soins critiques en temps réel

Mise à disposition de stocks spécifiques dans les maisons de retraite médicalisées selon une directive cantonale ; communication associée à cette distribution (y.c. mesures correctives)

Suivi rapproché des stocks de solutions hydroalcooliques

Gestion des pénuries de solutions hydroalcooliques ; communication des mesures mises en place

Contingentement des livraisons en fonction des consommations des services pour limiter le sur-stockage et le risque de vols

Récupération des flacons vides pour le remplissage à partir de la production interne mains

Présence quotidienne d'un pharmacien dans les unités de soins critiques

Revue de traitement de tous les patients hospitalisés pour le COVID19

Élaboration de documents d'assistance pharmaceutique nécessaires selon demandes des cliniciens et de la littérature ; communication de l'information

Maintien à jour d'une documentation clinique spécifique au COVID-19

Collaboration avec les spécialistes pour la mise en place de recommandations de traitement

Définition d'alternatives thérapeutiques ou d'administration en fonction des disponibilités des dispositifs médicaux (ex. pousses-
Responsabilité usuelle [oui/non/partiellement]

Non (nouvelle prestation mise en place spécifiquement pour la pandémie)

Non (nouvelle prestation mise en place spécifiquement pour la pandémie)

Non (nouvelle prestation mise en place spécifiquement pour la pandémie)

Non (nouvelle prestation mise en place spécifiquement pour la pandémie)

Non (habituellement cette responsabilité est déléguée au secteur Logistique pharmaceutique)

Partiellement (activité habituelle, mais renforcée)

Non (démarche propre à la situation)

Non (démarche propre à la situation)

Non (démarche propre à la situation)

Non (présence très nettement renforcée pendant la pandémie)

Partiellement (activité de revue renforcée pendant la pandémie et ciblée sur les patients COVID-19)

Oui (élaboration simplement renforcée et focalisée sur les problématiques rencontrées lors de la pandémie)

Partiellement (documentation ciblée sur les problématiques rencontrées lors de la pandémie)

Oui (activité simplement ciblée sur les traitements potentiels du COVID-19)

Non (démarche propre à la situation) 
Tableau I. Missions effectuées par les différents secteurs de la Pharmacie des hôpitaux de l'Est lémanique durant la première vague de pandémie COVID-19 au printemps 2020 (suite).

\begin{tabular}{|c|c|c|c|}
\hline Mission & Secteur & Exemple de mesures & $\begin{array}{l}\text { Responsabilité usuelle } \\
\text { [oui/non/partiellement] }\end{array}$ \\
\hline & & $\begin{array}{l}\text { seringues) et des médicaments ; } \\
\text { communication de l'information }\end{array}$ & \\
\hline \multirow[t]{2}{*}{$\begin{array}{l}\text { 6. Fabrication de } \\
\text { médicaments } \\
\text { individualisés }\end{array}$} & Fabrication & $\begin{array}{l}\text { Fabrication de formes non } \\
\text { disponibles de traitements potentiels } \\
\text { du SARS-CoV-2 (ex : suspension } \\
\text { d'hydroxychloroquine ou de lopinavir/ } \\
\text { ritonavir) }\end{array}$ & $\begin{array}{l}\text { Oui (fabrication simplement spécifique aux } \\
\text { besoins de la pandémie) }\end{array}$ \\
\hline & & $\begin{array}{l}\text { Mise en place de protocoles et de } \\
\text { kits de préparation de certains } \\
\text { médicaments injectables pour les } \\
\text { unités de soins (ex : tocilizumab) }\end{array}$ & $\begin{array}{l}\text { Oui (protocoles simplement spécifiques aux } \\
\text { besoins de la pandémie) }\end{array}$ \\
\hline \multirow[t]{2}{*}{$\begin{array}{l}\text { 7. Gestion des pharmacies } \\
\text { d'unité de soins }\end{array}$} & $\begin{array}{l}\text { Logistique } \\
\text { pharmaceutique }\end{array}$ & $\begin{array}{l}\text { Gestion des stocks de médicaments } \\
\text { directement dans les unités de soins }\end{array}$ & $\begin{array}{l}\text { Oui (gestion simplement intensifiée dans } \\
\text { les unités COVID-19) }\end{array}$ \\
\hline & & $\begin{array}{l}\text { Préparation des barquettes } \\
\text { journalières de médicaments des } \\
\text { patients }\end{array}$ & $\begin{array}{l}\text { Partiellement (effectué dans un site } \\
\text { hospitalier de plus qu'habituellement) }\end{array}$ \\
\hline $\begin{array}{l}\text { 8. Fourniture de masques } \\
\text { d'hygiène }\end{array}$ & $\begin{array}{l}\text { Logistique } \\
\text { pharmaceutique }\end{array}$ & $\begin{array}{l}\text { Distribution de stocks fédéraux de } \\
\text { masques d'hygiène pour les } \\
\text { professionnels de la santé pratiquant } \\
\text { en ambulatoire dans la région }\end{array}$ & $\begin{array}{l}\text { Non (distribution mise en place à la } \\
\text { demande du canton) }\end{array}$ \\
\hline
\end{tabular}

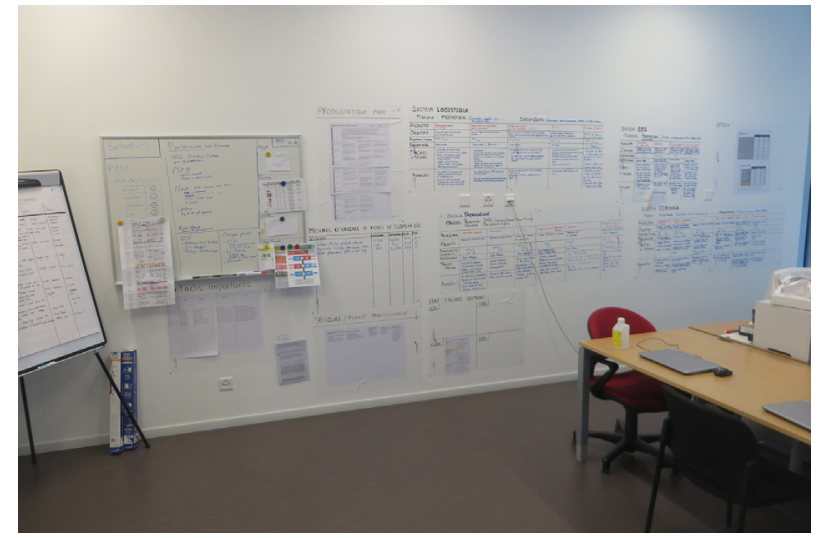

Figure 1. Salle de conduite de la cellule de crise de la pharmacie (crédit : N. Widmer, PHEL).

les ressources mobilisées pour cela ont nécessité de mettre en veilleuse diverses autres activités jugées moins prioritaires (Tableau III).

\section{DISCUSSION ET PROPOSITIONS}

Le recensement des missions effectuées par la PHEL durant la première vague de COVID-19 en Suisse démontre que les activités de cette pharmacie hospitalière ont été impactées à de multiples niveaux par cette situation extraordinaire. De nombreuses mesures et activités sortaient du cadre habituel d'une pharmacie interhospitalière. La communication interne et externe a également représenté un élément clef de la gestion de cette situation et de nouveaux types de points de situation intra- et intersecteur ont dû être organisés à horaires prédéterminés.

En priorité, la cellule de crise de la pharmacie, en collaboration avec les responsables de secteur de celle-ci, a dû garantir la continuité de ses ressources humaines en identifiant les fonctions clefs et les collaborateurs vulnérables et en priorisant l'engagement de chacun, tout en recourant autant que possible au télétravail lorsque celui-ci était possible. Un suivi attentif des effectifs a donc dû être mis en place, de même qu'un plan de protection associé à des mesures d'hygiène renforcées. Heureusement, seuls 4 collaborateurs sur 70 ont dû être isolés à domicile pour suspicion d'infection à SARSCoV-2 durant la période considérée dans cet article. Malgré les effectifs solides en personnel durant le printemps 2020, certaines missions hors des responsabilités usuelles de la pharmacie (fabrication de désinfectants pour les mains et distribution de masques d'hygiène aux professionnels de la santé de la région) n'ont pu être réalisées que grâce à l'appui d'appelés de la Protection civile.

En termes d'approvisionnement, la situation est rapidement devenue critique dès le mois de mars 2020, avec une pénurie de désinfectants pour les mains qui a nécessité la mise sur pied d'une production propre sur la base de la formule de I'OMS [25]. L'approvisionnement de plusieurs médicaments de soins critiques s'est progressivement compliqué jusqu'à miavril, bien qu'un accroissement préalable des stocks ait été réalisé au début du mois de mars, en prévision d'une augmentation de patients hospitalisés. Dans la phase ascendante de la pandémie en Suisse, ces réserves préventives n'ont cependant pas suffi à couvrir les besoins en certains produits, 
Tableau II. Médicaments spécifiques approvisionnés et problématiques associées.

\begin{tabular}{llll}
\hline $\begin{array}{l}\text { Classe } \\
\text { thérapeutique }\end{array}$ & Unités approvisionnées & Médicaments & Problématiques rencontrées \\
\hline Sédatifs & Soins critiques, urgences & Propofol & Proche de la rupture \\
& & \\
& & \\
& & \\
\end{tabular}

Soins palliatifs, urgences
$\begin{aligned} & \text { Soins critiques, soins } \\ & \text { palliatifs, maisons de } \\ & \text { retraite, urgences }\end{aligned}$

Dexmedetomidine

Kétamine

Étomidate

Clonazépam

Midazolam
Suivi rapproché des stocks

Proche de la rupture

Suivi rapproché des stocks

Proche de la rupture

Proche de la rupture

Alternatives identifiées au cas

où

Épargne du propofol $2 \%$ en favorisant l'utilisation du $1 \%$ en anesthésie

Sédation à base de kétamine et midazolam afin de garder le propofol pour les sevrages de sédation

Sevrage à la dexmedetomidine si plus de propofol disponible

Propofol ou clonidine

Midazolam, propofol, Dexmedetomidine

Kétamine, propofol

Midazolam ou levomepromazine

Kétamine, propofol,

dexmedetomidine

Pour les soins palliatifs en cas de rupture des benzodiazépines injectables : lorazepam, bromazepam, ou alprazolam par voie sublinguale ou buccogingivale

\section{Curares}

Soins critiques, urgences

Atracurium

Cisatracurium

Rocuronium

Suxaméthonium

Amines

Soins critiques, urgences

Noradrénaline

Antibiotiques

Soins critiques, médecine, Azithromycine maisons de retraite, urgences

\begin{tabular}{|c|c|c|}
\hline & & Ceftriaxone \\
\hline & & Co-amoxicilline \\
\hline & & $\begin{array}{l}\text { Pipéracilline/ } \\
\text { tazobactam }\end{array}$ \\
\hline \multirow[t]{3}{*}{ Antalgiques } & $\begin{array}{l}\text { Soins critiques, soins } \\
\text { palliatifs, urgences }\end{array}$ & Fentanyl \\
\hline & Soins critiques, urgences & Remifentanil \\
\hline & & Sufentanyl \\
\hline $\begin{array}{l}\text { Traitements } \\
\text { potentiels du } \\
\text { COVID-19 }\end{array}$ & $\begin{array}{l}\text { Soins critiques, médecine, } \\
\text { urgences }\end{array}$ & Hydroxychloroquine \\
\hline
\end{tabular}

Lopinavir/ritonavir

Remdesivir

Tocilizumab

Dexaméthasone
Proche de la rupture

Suivi rapproché des stocks

Suivi rapproché des stocks

Suivi rapproché des stocks

Proche de la rupture

Suivi rapproché des stocks

Proche de la rupture Suivi rapproché des stocks Suivi rapproché des stocks

Suivi rapproché des stocks

Suivi rapproché des stocks Suivi rapproché des stocks Suivi rapproché des stocks

Rupture, puis suivi rapproché des stocks

Rupture

Création de kit de reconstitution et protocole associé

Médicament pas suivi durant la première vague
Rocuronium en discontinu

Rocuronium en discontinu

Atracurium en continu

Dose majorée de rocuronium

Vasopressine à petite dose, adrénaline, dopamine

Autres dosages, clarithromycine

Autres dosages, co-amoxicilline

Autres dosages et fournisseurs

Autres dosages et fournisseurs

Remifentanyl, sufentanil, morphine

Hydromorphone et patchs de fentanyl en soins palliatifs

Fentanyl, sufentanyl, morphine Fentanyl, remifentanil, morphine Stock attribué par la Confédération

Stock attribué par la Confédération Aucune avant retour sur le marché Aucune 
Tableau II. Médicaments spécifiques approvisionnés et problématiques associées (suite).

\begin{tabular}{|c|c|c|c|c|}
\hline $\begin{array}{l}\text { Classe } \\
\text { thérapeutique }\end{array}$ & Unités approvisionnées & Médicaments & Problématiques rencontrées & $\begin{array}{l}\text { Alternatives identifiées au cas } \\
\text { où }\end{array}$ \\
\hline \multirow{7}{*}{$\begin{array}{l}\text { Traitements } \\
\text { supplémentaires } \\
\text { des maisons de } \\
\text { retraite }^{1}\end{array}$} & Maisons de retraite & $\begin{array}{l}\text { Ipratropium } \\
\text { (nébuliseur) }\end{array}$ & $\begin{array}{l}\text { Administration par } \\
\text { nébulisation et risques } \\
\text { associés pour le personnel }\end{array}$ & $\begin{array}{l}\text { Ipratropium aérosol doseur avec } \\
\text { chambre d'inhalation }\end{array}$ \\
\hline & & & Proche de la rupture & $\begin{array}{l}\text { Association fénotérol/ipratropium } \\
\text { aérosol doseur avec une chambre } \\
\text { d'inhalation }\end{array}$ \\
\hline & & $\begin{array}{l}\text { Salbutamol } \\
\text { (nébuliseur) }\end{array}$ & $\begin{array}{l}\text { Administration par } \\
\text { nébulisation et risques } \\
\text { associés pour le personnel }\end{array}$ & $\begin{array}{l}\text { Salbutamol aérosol doseur avec } \\
\text { chambre d'inhalation }\end{array}$ \\
\hline & & $\begin{array}{l}\text { Budésonide } \\
\text { (nébuliseur) }\end{array}$ & $\begin{array}{l}\text { Administration par } \\
\text { nébulisation et risques } \\
\text { associés pour le personnel }\end{array}$ & $\begin{array}{l}\text { Fluticasone aérosol doseur avec } \\
\text { chambre d'inhalation }\end{array}$ \\
\hline & & Paracétamol & Aucune & Pas nécessaire \\
\hline & & Acétylcystéine & Aucune & Pas nécessaire \\
\hline & & Morphine & Aucune & Pas nécessaire \\
\hline
\end{tabular}

${ }^{1}$ Mise à disposition d'un stock de réserve dans les établissements à la demande du canton de Vaud.

Tableau III. Activités principalement mises en suspens ou reportées durant la phase aiguë de la première vague de pandémie COVID-19.

\begin{tabular}{ll}
\hline Secteur & Activité \\
\hline Pharmacie dans son ensemble & Mise-à-jour de procédures et document dans le système de gestion de la qualité \\
& Accueil de stagiaires \\
& Certains projets de recherche \\
& Migration sur de nouveaux outils informatiques \\
\hline Direction & Conduite de certains projets \\
& Séances de groupes de travail et commissions \\
\hline Logistique pharmaceutique & Approvisionnements de divers services cliniques des hôpitaux partenaires ayant vu leur \\
& activité s'arrêter (consultations ambulatoires, chirurgie élective, etc.) \\
\hline Pharmacie clinique & Visites cliniques en présentiel dans les unités habituelles (transformées en analyse de \\
& dossier/conseils à distance) \\
& Enseignement de sujets hors-COVID-19 aux équipes médico-soignantes \\
& Enseignement présentiel aux étudiants en pharmacie (transformé en enseignement \\
& à distance) \\
Enseignement de sujets hors-COVID-19 aux équipes médico-soignantes (typiquement : projet \\
cantonal « Cercle de qualité ») \\
maisons de retraite & Projet informatique en lien avec la préparation automatisée des semainiers \\
\hline
\end{tabular}

déjà à risque de rupture d'approvisionnement avant les événements, comme dans de nombreux pays [26,27]. Les menaces de pénurie ont incité les pharmacies hospitalières suisses à s'entraider en mettant des médicaments et des solutions hydroalcooliques à la disposition des pharmacies qui en manquaient, étant donné que ni la Confédération ni les cantons ne proposaient d'aide concrète à ce moment-là. Par la suite, les autorités fédérales se sont efforcées de mettre sur pied avec l'Association suisse des pharmaciens de l'administration et des hôpitaux (GSASA) des mesures de coordination de cet approvisionnement à l'échelle nationale, impliquant même la pharmacie de l'armée. La mise en œuvre de ces mesures par les autorités fédérales auprès des fournisseurs de médicaments (libération anticipée de lots, importation, etc.), associé 
à une stabilisation des nouveaux cas, a permis de garantir un approvisionnement localement suffisant pour les hôpitaux approvisionnés par la PHEL, en évitant de justesse des pénuries de médicaments essentiels.

Sur le plan logistique également, la gestion du circuit du médicament au sein des nouvelles unités de soins créées pour l'occasion a représenté un défi important. Des assistant/es (préparateur/rices) en pharmacie ont dû changer du jour au lendemain d'activité et être affecté/es à ces nouvelles unités de soins ou à de nouvelles tâches (y.c. préparation, parfois, des traitements journaliers des patients). Dans ce contexte aussi, de nouvelles pharmacies d'unité de soins, dotées d'armoires informatisées sécurisées, ont dû être installées en un temps record au sein du Centre hospitalier de Rennaz. De façon générale, la présence de collaborateurs dans des unités de soins à risque, tant en hôpital qu'en maisons de retraite médicalisées, a nécessité l'application de mesures de protection adéquates. En maisons de retraite, la mise à disposition de médicaments spécifiques a d'ailleurs aussi constitué un enjeu au début de la pandémie. La liste de ceux-ci avait été définie par la Direction générale de la santé du canton de Vaud et ces médicaments devaient garantir une prise en charge directement dans l'établissement si nécessaire. Par la suite, la teneur de cette liste a été adaptée, se rapprochant plus d'une liste de prise en charge palliative, et les premiers emballages ont été entièrement fournis par le canton, les pharmacies de ville ou d'hôpital ne relayant que les suites de traitement.

Dans une période où de nouvelles données cliniques étaient publiées chaque jour sur la prise en charge des patients atteints de COVID-19 [27], un important travail de synthèse et de mise en forme de l'information médicale disponible a été réalisé par les pharmacien/nes clinicien/nes de la pharmacie. Ces spécialistes ont été quotidiennement en contact avec les médecins en charge des patients, que ce soit dans les unités de soins ou à distance, pour discuter tant de la prise en charge individuelle de ces derniers que des recommandations générales de traitement. L'identification d'alternatives aux principaux médicaments utilisés a aussi représenté une plus-value de la pharmacie, à l'image du travail d'autres pharmaciens cliniciens à travers le monde [26,28], et aurait permis une plus grande résilience en cas de pénurie avérée de traitements essentiels.

Enfin, le secteur Fabrication de la pharmacie, en plus de la production de désinfectants pour les mains en grande quantité (Fig. 2) et de ses activités habituelles, a également dû rapidement élaborer plusieurs protocoles de fabrication de médicaments indisponibles lorsque ces traitements étaient prescrits sous des formes galéniques spécifiques (comme des solutions orales d'hydroxychloroquine, médicament figurant encore dans les protocoles cliniques durant la période considérée). Des kits ont également pu être constitués afin de faciliter la préparation de certains traitements dans les unités de soins (ex : tocilizumab). Rattachée à ce secteur Fabrication, une unité dédiée à la gestion pharmaceutique des essais cliniques a également été rapidement constituée dans le cadre de l'étude Solidarity de l'OMS (visant à comparer plusieurs traitements supposément efficaces).

Sur la base de cette expérience exceptionnelle, le plan d'urgence de la pharmacie a été renforcé, ainsi que la formation associée du personnel. Diverses mesures d'anticipation sont en cours d'élaboration actuellement (ex : identification de tous les médicaments jugés indispensables en cas de

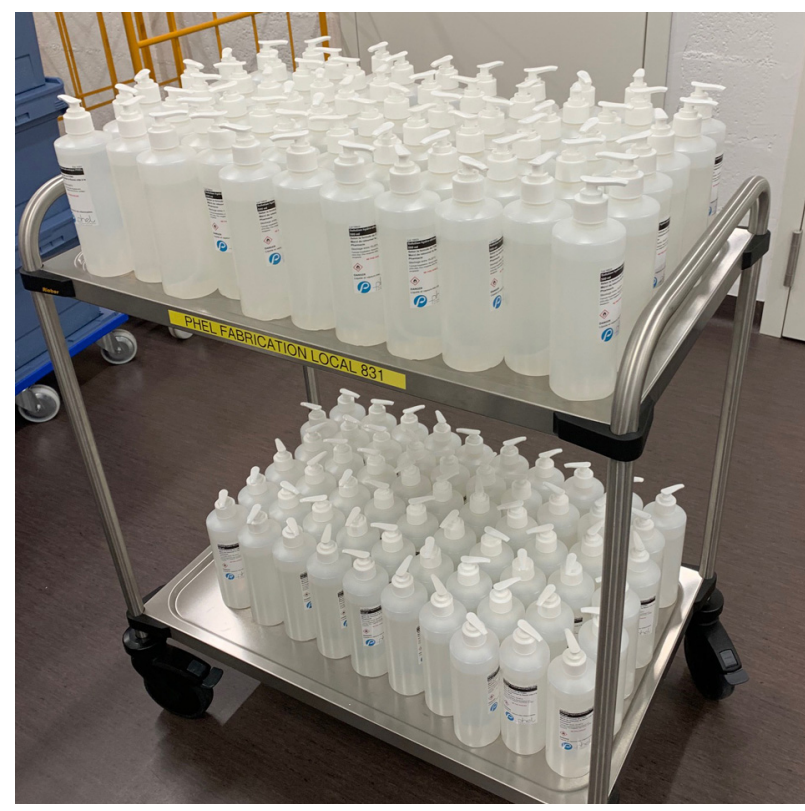

Figure 2. Production de solutions hydroalcoolique pour pallier aux pénuries rencontrées (crédit : S. Mösching, PHEL).

nouvelle vague épidémique, comme la dexaméthasone, et calibrage des stocks en conséquence, ainsi qu'optimisation de la gestion des ruptures et de leur prévention).

De façon plus globale, durant cette vague de pandémie COVID-19, une coordination ad hoc a été mise en place au niveau fédéral et cantonal pour faire face aux difficultés d'approvisionnement en médicaments. Cependant, cette coordination a été installée de façon relativement tardive par rapport à la cinétique de la pandémie. À plus long terme, il semblerait donc utile de mettre en place une coordination plus formelle en cas de crise entre les différents acteurs (autorités, fournisseurs, associations professionnelles, hôpitaux, pharmacie de l'armée), inspirée en partie de ce qui pouvait exister durant la guerre froide (service pharmaceutique coordonné) [29]. De plus, l'installation de cellules de crises entre hôpitaux d'une même région pourrait s'avérer utile pour mieux réguler les stocks de médicaments entre hôpitaux et échanger des idées pertinentes pour la gestion de la crise. Une telle cellule pourrait être animée par le biais de moyens télématiques.

\section{CONCLUSION}

L'analyse des événements présentée dans cet article montre que la pharmacie interhospitalière décrite a été confrontée à de nombreux défis durant la première vague de pandémie COVID-19 ayant touché la Suisse au printemps 2020. Certaines missions réalisées ont dépassé le cadre de ses responsabilités ordinaires. La majorité des pharmacies hospitalières suisses et européennes ont certainement fait face à des défis similaires, de même que celles situées dans d'autres continents au vu des récentes publications sur le sujet $[26,27,30]$. Des études systématiques à plus large échelle permettraient de tirer davantage de leçons de cette situation extraordinaire et 
de définir des mesures applicables à un collectif de pharmacies hospitalières plus grand en vue de la préparation à une nouvelle vague de pandémie, ainsi qu'à d'autres situations de catastrophes sanitaires. De façon plus générale, le rôle et les responsabilités des pharmaciens dans les systèmes de santé sont appelés à évoluer encore sur la base des leçons tirées de cette pandémie [27].

\section{Remerciements}

Le Département fédéral de la défense, de la protection de la population et des sports de la Confédération suisse, pour le financement du Centre spécialisé de pharmacie d'urgence et de catastrophe de l'Université de Genève (par le biais du Centre de compétences pour la médecine militaire et de catastrophe).

Tous les collaborateurs de la Pharmacie des Hôpitaux de l'Est lémanique (PHEL) pour leur engagement exceptionnel et leur solidarité durant cette crise, ainsi que Pascal Rubin, alors président de la PHEL, et Christian Moeckli, vice-président, pour leur soutien.

Déclaration de liens d'intérêts

Les auteurs déclarent ne pas avoir de liens d'intérêts.

\section{RÉFÉRENCES}

[1] Office fédéral de la santé publique (OSFP). Chiffres-clés des hôpitaux suisses 2018. Berne, Suisse: Confédération suisse; 2020.

[2] Office fédéral de la santé publique (OSFP). Politique nationale de la santé. Berne, Suisse: Confédération suisse; 2020, https://www. bag.admin.ch/bag/fr/home/strategie-und-politik/ nationale-gesundheitspolitik.html [cité le 29 juin 2020]

[3] Schoettker P, Potin M, Staeger P, Fuchs V, Yersin B, Ribordy V. La chaine des secours : le modele vaudois. Rev Med Suisse 2006;2(75):1849-53.

[4] Ordonnance sur le Service sanitaire coordonné (OSSC), du 27 avril 2005 (État le 1er février 2016) (RS 501.31). https:// www.admin.ch/opc/fr/classified-compilation/20041336/.

[5] Widmer N, Schumacher L, Meister T, Krähenbühl J-M, Mühlebach $\mathrm{S}$, Bonnabry P. Pharmacie d'urgence et de catastrophe : un centre spécialisé voit le jour en Suisse. pharmaJournal 2017;15:10-3.

[6] Oberholzer-Riss M. Unité de Doctrine UdD. Bull Info SSC 2016;1:72-8.

[7] Ordonnance sur l'approvisionnement économique du pays (OAEP), du 10 mai 2017 (État le $1^{\text {er }}$ juin 2017) (RS 531.11). Disponible : https://www.admin.ch/opc/fr/classified-compilation/ 20170044/index.html.

[8] Mühlebach S, Haudenschild U. Die Sicherstellung von Heilmitteln in der Schweiz bei Versorgungsengpässen: Die Rolle des Bereichs Heilmittel des Bundesamtes für Wirtschaftliche Landesversorgung in der Schweiz. Pharmind 2013;75(9):1461-5.

[9] Office fédéral de la protection de la population (OFPP). Catastrophes et situations d'urgence en Suisse : rapport technique sur la gestion des risques 2015. Berne, Suisse: Confédération suisse; 2015.

[10] Organisation de projet ERNS 14. Rapport final ERNS 14 : exercice du Réseau national de sécurité 2014 (ERNS 14). Berne, Suisse: Confédération suisse; 2015.

[11] Office fédéral de la protection de la population (OFPP). Umsetzungsbericht IV zu den Empfehlungen aus der Sicherheitsverbundübung SVU 14. Berne, Suisse: Confédération suisse; 2019.
[12] Campbell P. Peste noire (1347-1352) et Covid-19 (2019-20-?) 1. Les similitudes. Med Catastrophe Urg Collectives 2020;4(2):14951.

[13] Huang C, Wang Y, Li X, Ren L, Zhao J, Hu Y, et al. Clinical features of patients infected with 2019 novel coronavirus in Wuhan, China. Lancet 2020;395(10223):497-506.

[14] Hui DSC, Zumla A. Severe acute respiratory syndrome: historical, epidemiologic, and clinical features. Infect Dis Clin North Am 2019;33(4):869-89.

[15] Rajgor DD, Lee MH, Archuleta S, Bagdasarian N, Quek SC. The many estimates of the COVID-19 case fatality rate. Lancet Infect Dis 2020;20(7):776-7.

[16] Gatto M, Bertuzzo E, Mari L, Miccoli S, Carraro L, Casagrandi $\mathrm{R}$, et al. Spread and dynamics of the COVID-19 epidemic in Italy: effects of emergency containment measures. Proc Natl Acad Sci U S A 2020;117(19):10484-91.

[17] Worldometers.info. Coronavirus Update (Live). Dover, Delaware, USA: Dadax LLC; 2020, https://www.worldometers.info/ coronavirus/ [cité le 12 mars 2020].

[18] Ordonnance 2 sur les mesures destinées à lutter contre le coronavirus (COVID-19) (Ordonnance 2 COVID-19), du 13 mars 2020 (État le 20 juin 2020) (RS 818.101.24). Disponible : https://www. admin.ch/opc/fr/classified-compilation/20200744/.

[19] Loi fédérale sur la lutte contre les maladies transmissibles de I'homme (Loi sur les épidémies, LEp), du 28 septembre 2012 (État le 25 juin 2020) (RS 818.101). Disponible : https://www.admin.ch/ opc/fr/classified-compilation/20071012/.

[20] Droz R. Opération CORONA 20. Rev Mil Suisse 2020;4:5-7.

[21] Salzer R. SARS-CoV-2 Cases communicated by Swiss Cantons and Principality of Liechtenstein (FL). Statistische Amt des Kanton Zürichs. Zürich, Suisse; 2020, https://rsalzer.github.io/ COVID_19_CH/ [cité le 13 août 2020].

[22] Bureau régional de I'Organisation mondiale de la santé pour I'Europe (WHO/Europe). WHO COVID-19 Health System Response Monitor: Switzerland. Copenhagen, Denmark: OMS/ Europe; 2020, https://www.covid19healthsystem.org/countries/ switzerland/ [cité le 29 juin 2020].

[23] Maudet L, Sarasin F, Dami F, Carron PN, Pasquier M. Urgences prehospitalieres : crise COVID-19. Rev Med Suisse 2020;16 (691-2):810-4.

[24] Ageron FX, Sarasin F, Pasquier M, Carron PN. Urgences hospitalieres : crise COVID-19 et aspects organisationnels. Rev Med Suisse 2020;16(692):924-9.

[25] Organisation mondiale de la santé (OMS). Guide de Production locale: Formulations des produits hydro-alcooliques recommandés par l'OMS. Genève, Suisse: OMS; 2000.

[26] Gross AE, MacDougall C. Roles of the clinical pharmacist during the COVID-19 pandemic. J Am Coll Clin Pharm 2020;3(3):564-6.

[27] Bragazzi NL, Mansour M, Bonsignore A, Ciliberti R. The role of hospital and community pharmacists in the management of COVID-19: towards an expanded definition of the roles, responsibilities, and duties of the pharmacist. Pharmacy 2020;8(3):140.

[28] Siow WT, Tang SH, Agrawal RV, Tan AYH, See KC. Essential ICU drug shortages for COVID-19: what can frontline clinicians do? Crit Care 2020;24(1):260.

[29] Kratzer B. Der Koordinierte Pharmazeutische Dienst. Schweizer Spital 1987;8:38-9

[30] Liu S, Luo P, Tang M, Hu Q, Polidoro JP, Sun S, et al. Providing pharmacy services during the coronavirus pandemic. Int J Clin Pharm 2020;42(2):299-304 